\title{
DIAGNÓSTICO SITUACIONAL DA ATUAÇÃO DOS CENTROS DE INFORMAÇÃO SOBRE MEDICAMENTOS NO BRASIL
}

Situational diagnosis of the performance of drug information centers in Brazil

Submetido em: 05/08/2016 Aceito em: 07/12/2016

Publicado em: 30/03/2017

Diagnóstico de la situación de la actuación de los centros de información sobre

Maria Aparecida Nicoletti ${ }^{\text {* }}$, Gabriela Rodrigues da Silva Marques',

Patricia Melo Aguiar', Sílvia Storpirtis ${ }^{1}$

1 Farmácia Universitária, Departamento de Farmácia, Faculdade de Ciências Farmacêuticas,

Universidade de São Paulo

*E-mail: nicoletti@usp.br

\section{Resumo}

O repasse da informação de qualidade mostra-se atualmente como uma das principais ferramentas para o estabelecimento do uso racional de medicamentos. Diante de um cenário no qual se consolidam inúmeras fontes de dados e de conhecimento, de caráter científico ou não, é urgente a necessidade de análise da informação antes de sua aplicação farmacoterapêutica. Os Centros de Informação sobre Medicamentos (CIM) constituem uma importante ferramenta para a obtenção de informação sobre medicamentos, que poderá ser disponibilizada a diferentes públicos alvo. Foi realizada análise qualiquantitativa, descritiva e transversal, no período de dezembro de 2013 a março de 2014, envolvendo um elenco de 27 centros e serviços de informação sobre medicamentos brasileiros. Observou-se neste estudo que o número de CIM brasileiros é oscilante ao longo dos anos, muito embora seja crescente a iniciativa de instalar centros de informação de medicamentos pelo território nacional. Entretanto, os CIM precisam suportar entraves de diversas naturezas para a promoção de suas atividades, o que acarreta, na maioria dos casos, o não cumprimento de suas propostas iniciais, isto é, a prática de informação passiva em um tempo não satisfatório à necessidade da informação.

Palavras-chave: Medicamentos, informação, Centro de Informações em Saúde.

\section{Abstract}

The quality of currently transfer information shows up as one of the main tools for establishing the rational use of medicines. Faced to a scenario where consolidate numerous sources of data and knowledge, scientific or not, there is an urgent need for information analysis prior to their pharmacotherapeutic application. The Drug Information Center (DIC) constitutes an important tool for obtaining information about drugs, which may be available to different target audiences. Qualitative and quantitative, descriptive and cross-sectional analysis was conducted from December 2013 to March 2014, involving a cast of 27 centers and information services considering Brazilian medicines. The study shows that the number of Brazilians DIC is oscillating over the years, although it is growing the initiative to perpetuate the application of drug information centers throughout the country. However, the DIC units must endure barriers of various kinds to promote their activities, contributing to, in most cases, non-compliance with their original proposals, that is, and the practice of passive information in a time not satisfying the needs for information.

Keywords: Medications, information, Health Information Center. 


\section{Resumen}

En la actualidad, el repase de informaciones de calidad se presenta como una de las principales herramientas para establecer el uso racional de los medicamentos. Frente a un escenario en el que se consolidan innúmeras fuentes de datos y de conocimientos de carácter científico, o no, urge la necesidad de análisis de la información antes de su aplicación farmacoterápica. Los Centros de Información de Medicamentos (CIM) son una herramienta importante para obtener información acerca de los medicamentos, que podrá estar disponible para los diferentes grupos sociales. Se hizo un análisis cualitativo y cuantitativo, descriptivo y transversal entre diciembre 2013 y marzo 2014, con la participación de un elenco de 27 centros y servicios de información sobre medicamentos brasileños. En ese estudio se observó que el número de CIM brasileño está oscilando en los últimos años, a pesar de la creciente iniciativa de instalar centros de información de medicamentos en todo el país. Sin embargo, los CIM deben enfrentar barreras de diversas índoles para promover sus actividades, lo que origina en grande parte de los casos, el incumplimiento de sus propuestas iniciales, es decir, la práctica de información pasiva en un momento en el que la información no responde a las necesidades sociales.

Palabras clave: Medicamentos, información, Centro de Informaciones de Salud.

\section{INTRODUÇÃO}

A Organização Mundial da Saúde estabelece como uso racional de medicamentos (URM) a situação em que o paciente recebe um medicamento apropriado à sua condição clínica, em doses adequadas às suas necessidades individuais, em um período satisfatório e ao menor custo para si e para a comunidade. Contudo, mesmo sendo uma definição estabelecida em 1985, na Conferência Mundial Sobre o Uso Racional de Medicamentos, em Nairóbi(1), erros de medicação ainda são recorrentes em cenário nacional. Equívocos nas práticas de prescrição, administração e dispensação dos medicamentos podem acarretar danos graves em pacientes ${ }^{(2)}$ como, muitas vezes, internações hospitalares e/ou óbito. Assim, o uso racional de medicamentos (URM) mostra-se como uma estratégia urgente a ser aplicada em território nacional para a promoção da saúde. Em 2002, a OMS definiu 12 estratégias para a implementação do URM. Entre elas, estão os serviços prestados pelos Centros de Informação sobre Medicamentos $^{(3)}$, que poderão auxiliar grandemente no fornecimento de informação técnico-científica tanto à comunidade científica, para ajudar na tomada de decisões, como, também, à comunidade leiga, no que tange ao conhecimento sobre o medicamento e o seu uso correto.

Diante do desenvolvimento da indústria farmacêutica e do avanço científico nas últimas décadas, muita informação sobre terapias medicamentosas pode ser conseguida por vários meios. Assim, tornou-se um desafio para os profissionais de saúde fazer bom uso da informação diante de tantas fontes e referências muitas vezes de credibilidade discutível. A informação deverá estar alicerçada na comprovação de evidência em saúde para que possa ser utilizada com segurança. Portanto, a sua disponibilização centralizada poderá interferir positivamente na redução de erros evitáveis.

Da necessidade de informação de boa qualidade, surgiu a criação do Centro de Informação sobre Medicamentos (CIM).-O primeiro CIM nasceu em 1962, na Universidade de Kentucky, nos Estados Unidos da América do Norte ${ }^{(4)}$. Após a sua criação, a iniciativa se disseminou para a Europa, chegando ao Brasil em 1994, com o Centro Brasileiro de Informação sobre Medicamentos 
(Cebrim), criado pelo Conselho Federal de Farmácia (CFF) e atuante até o momento(5). Em 1999, foi estabelecida uma rede integrada de CIM brasileiros, denominada Sistema Brasileiro de Informação sobre Medicamentos (Sismed)(6), regida mediante um protocolo de cooperação, que define os critérios para o estabelecimento dos centros, além de atividades de cooperação entre os CIM. As ações do Sismed colaboraram com a criação da Rede Brasileira de Centros e Serviços de Informação sobre Medicamentos (Rebracim), organizada em 2010 com o apoio da Organização Pan-Americana da Saúde e do Ministério da Saúde. A Rebracim foi oficialmente institucionalizada no Sistema Único de Saúde por meio da Portaria Ministerial n 2.647 de 2013 e tem o objetivo de coordenar uma rede de centros e serviços de informações sobre medicamentos e promover o uso racional de medicamentos ${ }^{(7)}$.

Conceitualmente, o CIM é o local onde se reúnem, analisam, avaliam e fornecem informações sobre medicamentos, visando a seu uso racional(6). O Serviço de Informação sobre Medicamentos (SIM) é comumente usado como sinônimo de CIM, mas o serviço difere do centro quanto aos recursos, à estrutura e ao alcance de seus serviços. O SIM atende à demanda interna de uma instituição, como um hospital, e o CIM tem maior abrangência, atuando como referência em um país, uma região ou um estado(6). Outro mecanismo de informação sobre medicamentos é aquele que as indústrias farmacêuticas disponibilizam aos consumidores e profissionais da saúde, denominado de Serviço de Atendimento ao Consumidor (SAC), que trata de assuntos relacionados aos produtos de sua linha, sendo, portanto, bem específico, além de envolver esclarecimentos e registros de desvio de qualidade de seus produtos que não necessariamente estarão diretamente relacionados à ação do medicamento(8).

Os CIM/SIM, diferentemente das bibliotecas e dos centros de documentação, não proporcionam apenas documentos ou referências bibliográficas, mas soluções para problemas concretos sobre medicamentos ou para uma situação clínica de um paciente. Prestam informação selecionada, processada e avaliada por profissionais especializados, focalizada na necessidade particular do solicitante mediante um serviço altamente custo-efetivo ${ }^{(9,10)}$.

A informação técnico-científica é necessária para subsidiar decisões na área da saúde. É preciso agilidade no processo de sua disponibilização e ela deve estar baseada em evidência científica. Configura-se, assim, uma necessidade para o uso racional de medicamentos.

A Saúde Baseada em Evidências (SBE) é uma abordagem que utiliza as ferramentas da Epidemiologia Clínica, da Estatística, da Metodologia Científica e da Informática para trabalhar a pesquisa, o conhecimento e a atuação em saúde, com o objetivo de aplicar a melhor informação disponível para a tomada de decisão nesse campo. De modo geral, a SBE busca promover a integração da experiência clínica às melhores evidências disponíveis, considerando a eficácia, a efetividade, a eficiência e a segurança das intervenções em saúde ${ }^{(11)}$.

Quanto à infraestrutura, um CIM/SIM deve preencher, pelo menos, dois requisitos: contar com um farmacêutico especialista em informação sobre medicamentos e ter bibliografia sobre medicamentos reconhecida internacionalmente e a mais atualizada possível(6). Um CIM/SIM pode prover a informação de diferentes formas, como a informação passiva ou reativa, a qual é oferecida em resposta à pergunta de um solicitante. Além disso, pode promover a informação ativa, ou seja, atividades de sua própria iniciativa que repassam informações sobre medicamentos, entre elas publicação de boletins e informes, suporte à Comissão Farmacoterapêutica (CFT), ações em farmacovigilância, ensino, entre outras ${ }^{(6,8)}$.

O objetivo deste estudo é fazer um levantamento das unidades de CIM/SIM presentes no território nacional, no período de dezembro de 2013 a março de 2014, quanto ao funcionamento e à sua estrutura, considerando a necessidade da disponibilização de fontes de informação técnico-científica baseada no uso racional de medicamentos. 


\section{MATERIAL E MÉTODOS}

Trata-se de um estudo, descritivo e transversal, realizado no período de dezembro de 2013 a março de 2014, considerando o elenco de 27 centros e serviços de informação sobre medicamentos no Brasil, segundo o Conselho Federal de Farmácia. Também foi realizada uma pesquisa na web, utilizando os seguintes termos de busca: CIM, Centro de Informação de Medicamento, Centro de Informação sobre Medicamento, Serviço de Informação de Medicamento e SIM.

Foi elaborada uma lista de unidades de CIM/SIM brasileiros que consistiu, basicamente, na junção de dados fornecidos tanto pelo site do Conselho Federal de Farmácia( ${ }^{(4)}$ e pelo elenco descrito pela Rebracim(12), além de pesquisas dos centros via internet.

Dentre as funções desempenhadas por um CIM/SIM, a principal é a provisão da informação passiva. Para avaliar esse aspecto, foi realizado o encaminhamento de solicitação de informação às unidades mapeadas de CIM/SIM, a partir de dois tipos:

O primeiro tipo envolveu um quadro de saúde aleatório apresentado por um paciente hipotético, sendo encaminhada a seguinte situação ao endereço eletrônico de acesso:

“Realizando estudo de caso em paciente idoso, submetido à polifarmácia, gostaria de saber o que a literatura aponta para a interação medicamentosa entre Lasix (Furosemida) e Dimefor (Cloridrato de Metformina).

Dados do paciente:

Idade: 74 anos

Sexo: masculino

Peso: $82 \mathrm{~kg}$

Altura: $1,71 \mathrm{~m}$

No aguardo,

Grata".

Para o segundo, foi enviada uma solicitação de informação aleatória, sem paciente envolvido, apenas sobre os efeitos colaterais do bromidrato de fenoterol, para a verificação de como o CIM/SIM poderia estruturar o questionamento enviado.

No momento da solicitação, foi verificado se os CIM/SIM disponibilizavam formulário específico em site para a submissão da pergunta ou se esta era realizada via e-mail. Deve-se ressaltar que, neste estudo, solicitações realizadas por telefone não foram objeto de análise.

Quanto às respostas das solicitações, analisou-se o tempo para o seu envio, bem como o layout destas, ou seja, se os CIM/ SIM optaram pela elaboração de um documento ou por um texto informal. Ademais, a obtenção de uma resposta de solicitação é um dos indicativos de que um CIM/SIM se encontra ativo. Assim, utilizou-se desse dado, bem como de ligações telefônicas no caso das unidades que não responderam às solicitações - para se obter a porcentagem de centros e serviços ativos no país.

Um CIM/SIM pode, também, promover a informação ativa. A análise desse tipo de informação se deu pelo estabelecimento de contato, via telefone e e-mail, com os centros, solicitando a eles informações a respeito das atividades de informação ativa prestada. Além disso, o estudo destes serviços, entre outros aspectos gerais, como afiliação, também foi realizado por meio de visita aos sites de tais centros, o que permitiu a análise de documentos de campanhas e artigos, particularmente no caso dos CIM em que não foi possível, via contato direto (e-mail ou telefone), inteirar-se da informação ativa prestada. Ainda em relação à afiliação, ressalta-se que os hospitais universitários foram classificados como afiliados a universidades e não a hospitais. 


\section{RESULTADOS E DISCUSSÃO}

Os Centros de Informação sobre Medicamentos podem atuar de duas maneiras, ou seja, com o desenvolvimento de informação passiva (questionamento sobre a farmacoterapia relacionada a determinado paciente) ou com a informação ativa (que pode ser representada por atividades de educação e difusão da informação sobre medicamentos). Saliente-se que a informação passiva poderá ser realizada por telefone, correio, correio eletrônico ou pessoalmente ${ }^{(6)}$.

\section{Informação passiva}

Neste estudo, constatou-se que, em sua maioria, os CIM/SIM brasileiros direcionam o serviço de informação passiva para os profissionais de saúde, chegando, em alguns casos, a exigir o número de registro profissional para que o questionamento possa ser efetivado. Foi observado também que, ainda no que tange a restrições, dois centros $(7,40 \%)$ atendem apenas solicitações advindas de seu estado. Por se tratar de instituições localizadas externamente ao estado de São Paulo, localidade onde este estudo se realizou, não foi possível analisar a informação passiva de tais centros.

Este cenário nos indica que os CIM/SIM brasileiros não estão voltados diretamente à população leiga, restrição esta que aparenta estar ligada ao fato de que muitos centros estão ainda se estabelecendo e necessitam restringir seu público, limitando, assim, o número de casos a serem estudados e comprometendo a variabilidade de situações-problema contidas em seu banco de informações.

A Europa compreende a importância do Serviço de Informação (SI) proveniente da população leiga no processo de enriquecimento do banco de dados dos CIM. A Grã-Bretanha é um exemplo de apoio à atuação do CIM no suporte à população leiga, além de profissionais da saúde ${ }^{(10)}$.

Também considerando a situação de outros países, em outro continente como o asiático, em relação a centros de informação sobre medicamentos, a informação obtida a partir do Drug Information and Research Center ${ }^{(14)}$ (DIRC - que foi o primeiro CIM criado na Índia, em 1997) indicou que este destina seus serviços diretamente ao paciente, sanando dúvidas (sobre posologia, efeitos adversos, entre outras) e informando-os. Trata-se, assim, de uma demonstração prática da aplicação de um centro de informação de medicamentos voltado à promoção do URM.

Em relação à qualidade dos serviços prestados via informação passiva, o primeiro critério observado foi a via de recepção das SI oferecidas em cada centro, considerando se esta respeitava uma padronização ou apresentava caráter informal. Observou-se que 21 dos 27 centros $(77,78 \%)$ recebiam suas perguntas via e-mail, constatando-se, portanto, a ausência de uma padronização específica (uma vez que esta via de acesso não obedece a um formato previamente estabelecido), entre outras características que, provavelmente, dificultam o processo de documentação da questão enviada e comprometem a construção de um banco de dados.

Foi observado que alguns CIM/SIM disponibilizavam formulários que exigiam informações demasiadamente detalhadas, restringindo, muitas vezes, solicitações de caráter simplificado, ou seja, aquelas que podem não envolver paciente e/ou polifarmácia. Indica-se, portanto, como ideal, um formulário que seja passível de dar o suporte a qualquer tipo de solicitação, seja ela envolvendo paciente ou não, sem requerimento de informações obrigatórias, uma vez que estas podem confundir o solicitante e demandar um tempo a mais que o necessário para a sua elaboração.

O equacionamento de informação praticado por um CIM/SIM só é válido se aplicado de forma efetiva à sua finalidade. Ou seja, se a informação técnico-científica requerida tem qualidade e é fornecida em um tempo adequado, já que trata-se de uma 
ferramenta valiosa para a tomada de decisão. Pelos dados obtidos, 14 das 25 unidades brasileiras de CIM/SIM (51,85\%) não retornaram à solicitação de informação em um período de três meses (Figura 1).

Figura 1. Tempo de resposta às solicitações encaminhadas ao elenco de 27 CIM/SIM brasileiros durante o período de dezembro de 2013 a março de 2014.

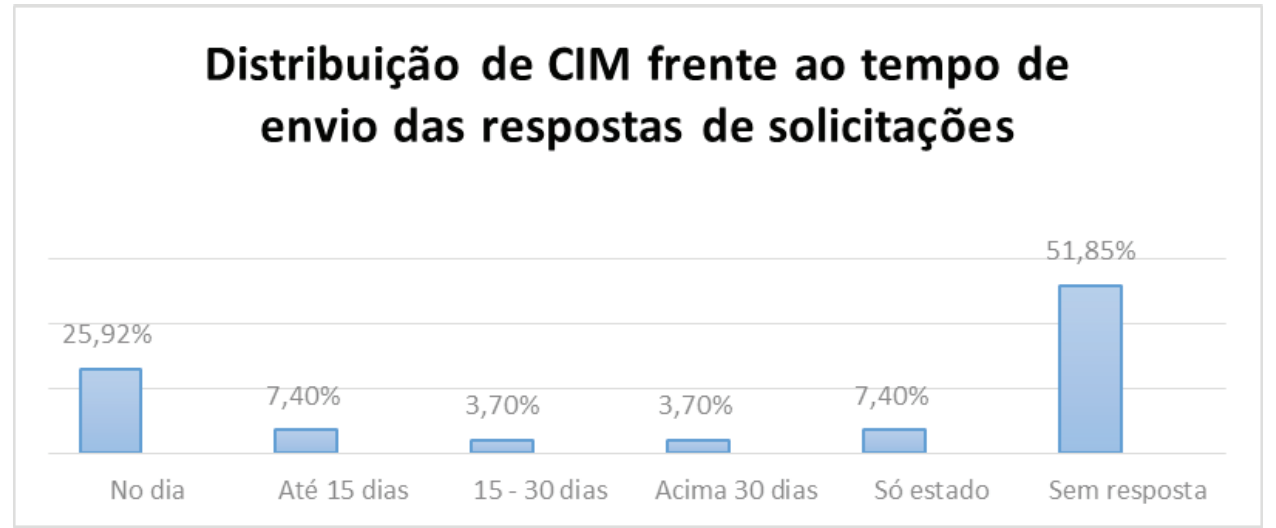

Considerando-se a resposta de SI elaborada pelos centros, a informalidade também se mostrou evidente. Observou-se que cerca de $55 \%$ das respostas das SI eram de textos enviados por e-mails, sem padronização específica inerente a um documento (Figura 2). Apenas em $45 \%$ das respostas foi verificada uma elaboração apropriada em arquivo em extensão pdf, com código de controle da solicitação, o que dificulta possíveis alterações do texto e facilita consultas futuras, respectivamente. A falta de padronização frequentemente observada nas vias de submissão, bem como as respostas das SI, indica uma informalidade predominante que poderá comprometer o enriquecimento do banco de dados de cada centro.

Figura 2. Perfil de vias de submissão e resposta de solicitação de informação levantado a partir de solicitações de informação enviadas a 27 CIM/SIM brasileiros durante o período de dezembro de 2013 a março de 2014.

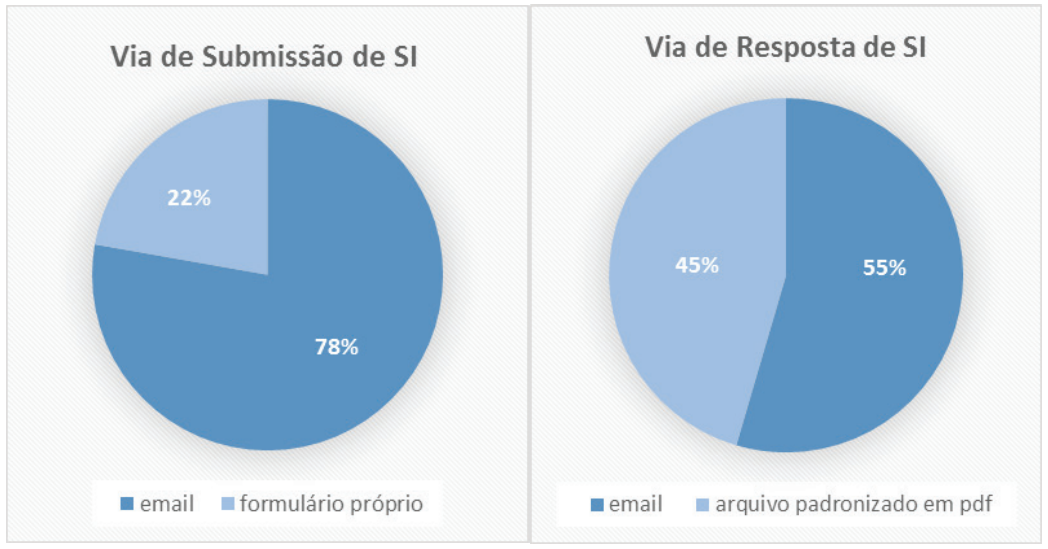


Considerando o fato de que a maioria dos CIM/SIM não respondeu às solicitações (Figura 1), e para assegurar se realmente estavam inoperantes, tais centros foram contatados por telefone, a fim de se certificar sua real condição no momento. $\mathrm{O}$ resultado obtido foi de que apenas um pouco mais da metade (55\%) dos CIM/SIM nacionais estão comprovadamente operantes, pelos critérios utilizados neste estudo (encaminhamento de e-mail, contato por telefone, consulta no website). Nenhum tipo de contato foi possível de ser estabelecido, no período de três meses, com a outra parcela de $45 \%$. Assim, pode ser inferido que as listagens de centro de informação sobre medicamentos nem sempre apresentam atualização. Até porque são necessários recursos financeiros para a continuidade dos trabalhos que envolvem recursos humanos, sistema de tecnologia em informação continuamente atualizado e acesso a bases científicas de dados, pagas em sua grande maioria, o que muitas vezes se configura um ônus que impossibilita a continuidade de manutenção desses centros, tornando-os inoperantes.

Quando se estabelecia contato, por inúmeras vezes, não havia farmacêutico disponível para sanar dúvidas. A disponibilidade de profissionais tecnicamente qualificados é problema no cenário atual porque exige custo. Uma unidade relatou ainda que estava temporariamente fechada por problemas de espaço físico. De fato, os centros brasileiros enfrentam inúmeros problemas, desde respaldo financeiro ao espaço físico, para a manutenção de seus serviços. Abaixo, encontra-se, comparativamente, a distribuição geográfica dos CIM/SIM brasileiros pertencentes à Rebracim (filiados a instituições públicas e filantrópicas), em estudo realizado por Vidotti, em 1999(6); e também em relação à distribuição observada neste estudo de unidades públicas e particulares, ativas em território brasileiro, conforme Figura 3.

Figura 3. llustrações comparando o elenco de CIM brasileiros do ano de 1999, exposto por Vidotti et al 2000, e o elenco de 27 CIM/SIM ativos e inativos, obtido no presente trabalho, durante o período de dezembro de 2013 a março de 2014.

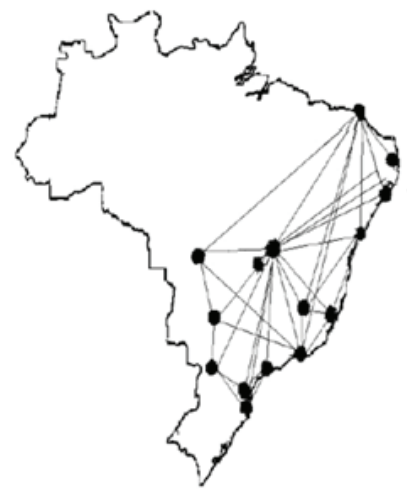

ELENCO SISMED 1999

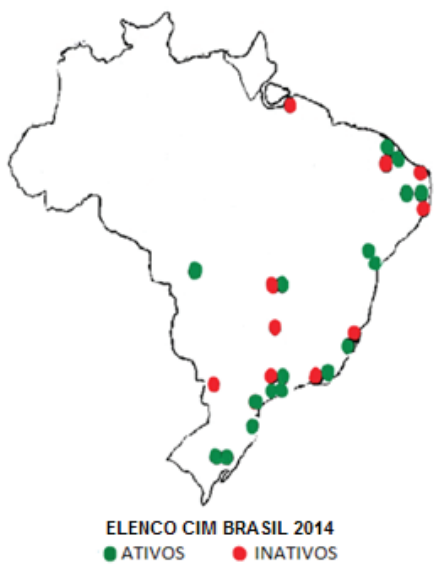

- ATIVOS INATIVOS

\section{Informação ativa}

Em relação às atividades em informação ativa, considerou-se a análise deste parâmetro apenas nas unidades comprovadamente ativas. A Figura 4 evidencia que as atividades principais desempenhadas pelos centros são a publicação de boletins e informes, juntamente com as atividades de ensino, seguidas pelo suporte à Comissão de Farmácia e Terapêutica. 
Figura 4. Distribuição de tipos de atividades em informação ativa realizadas pelo elenco brasileiro de 27 CIM/SIM analisados no presente trabalho durante dezembro de 2013 e março de 2014.

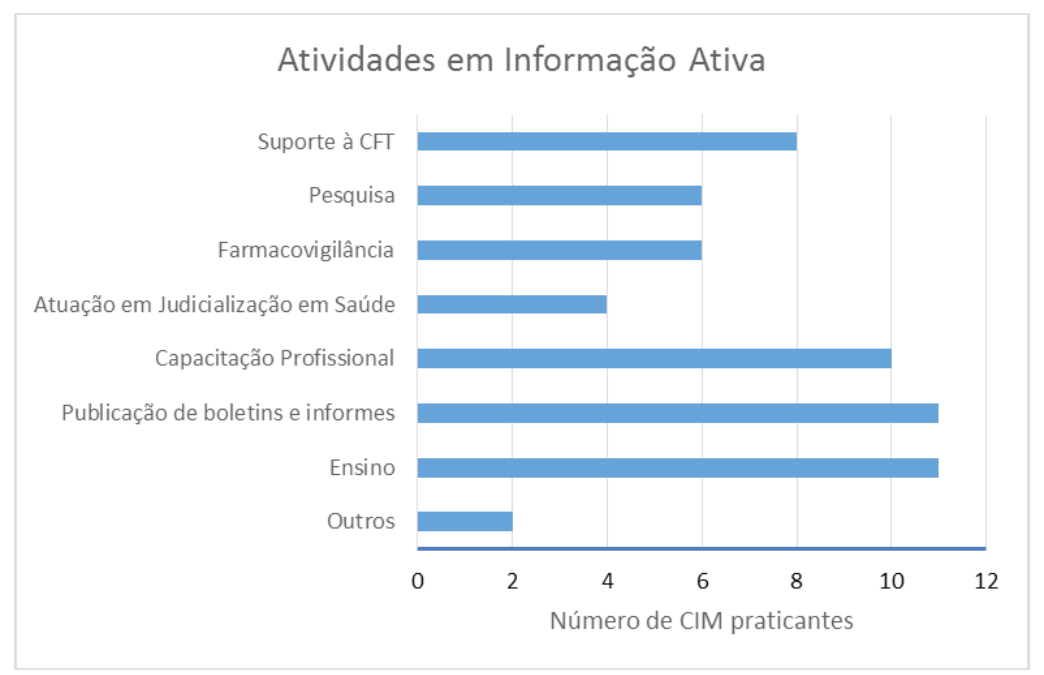

\section{Afiliação}

Diante do inconstante elenco de unidades de CIM/SIM nacionais, em decorrência da falta de recursos financeiros ou até mesmo de espaço físico, é conveniente a análise da afiliação de cada um deles. De maneira geral, a iniciativa acadêmica afilia os CIM/ SIM brasileiros (considerando as parcelas Universidades e Hospitais Universitários, que detêm 50\% do número de afiliações), conforme Figura 5.

Figura 5. Perfil e afiliação do elenco brasileiro de 27 CIM/SIM analisado no período de dezembro de 2013 a março de 2014.

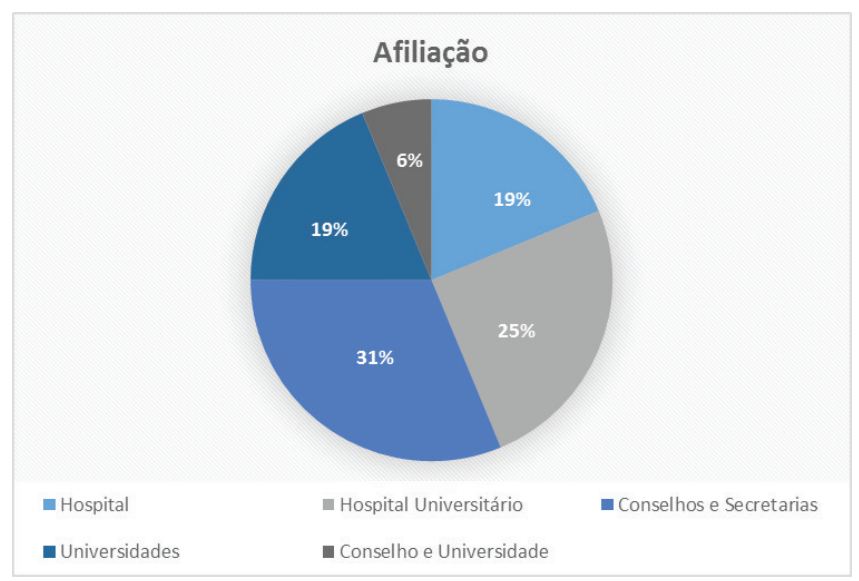


Visivelmente, o expressivo número de atividades de informação proativa em ensino se correlaciona com a detenção de afiliações por iniciativa acadêmica. Apesar do crescente número de unidades em instituições privadas (principalmente hospitais particulares), as instituições de ensino são promotoras do conceito CIM/SIM em território nacional, utilizando as atividades desenvolvidas em trabalhos de pesquisa e ensino, condição esta facilmente compreensível pela natureza da atividade.

O repasse de informação só é válido se aplicado de forma efetiva à terapêutica, afinal, uma resposta de SI demorada contradiz as circunstâncias de criação de um CIM/SIM, uma vez que afeta um dos pilares do URM - o tempo - e o CIM/SIM deve ser instrumento de otimização da informação técnico-científica. As unidades precisam suportar entraves de diversas naturezas para a promoção e a continuidade de suas atividades, contribuindo para, na maioria dos casos, o não cumprimento de suas propostas iniciais, isto é, a prática de informação passiva, em um tempo não correspondente à realidade.

Os CIM/SIM, de maneira geral, ainda padecem de imaturidade sob alguns aspectos. Boa parcela restringe seu atendimento à equipe multiprofissional em saúde, ainda que seja conhecida a importância de expansão de atendimento para enriquecer a base de dados e as experiências em ensino. O tratamento informal dado à prática de informação passiva, bem como a demora no seu repasse, pode ser resultante do fato de que muitas unidades ainda estão se estabelecendo, utilizando um modelo provisório, não estável para a realização de suas atividades.

Assim, mais do que a ciência da importância do CIM/SIM no contexto da saúde e na prática de ensino (evidenciada nos resultados em atividade proativa), é necessário prover as condições para o seu estabelecimento, incentivadas tanto por meio de fontes de financiamento, capacitação de profissionais qualificados, deliberação de um espaço físico adequado, quanto por suas estruturas intrínsecas, como computadores, entre outras. Uma vez bem estabelecidos, por meio de um modelo convenientemente estruturado, os centros de informação sobre medicamentos podem contribuir diretamente para intervenções diretas em saúde pública. Um acompanhamento via informações ativa e passiva, dos perfis de uso de medicamentos demandados pelos sistemas públicos, pode beneficiar ainda o Sistema Único de Saúde, o qual terá condições de manter um maior controle do dispêndio de medicamentos e, consequentemente, dos gastos, além de criar seu banco de dados e direcionar atividades que resultem em educação em saúde ${ }^{(8)}$.

\section{CONCLUSÃo}

A iniciativa da criação e funcionamento de um CIM/SIM é válida e muito poderá acrescentar na formação acadêmica, considerando a oportunização às fontes de informação sobre medicamentos, além de todos os procedimentos relacionados ao fluxo da estruturação de resposta adequada e cientificamente válida, o que, certamente, resultará em benefícios à saúde pública. Pelos dados levantados referentes ao período de tempo estudado, todavia, a rede nacional de centros de informação de medicamentos ativos, que disponibiliza, também, informação à população, carece de recursos e merece a atenção das instituições públicas e privadas para que seja um serviço que tenha continuidade, além de se constituir uma ferramenta de educação em saúde.

\section{AGRADECIMENTOS}

À Pró-Reitoria de Graduação da Universidade de São Paulo e à Farmusp, pelo apoio concedido. 


\section{REFERÊNCIAS}

1. WHO. World Health Organization. The rational use of drugs: report of the conference of experts. Nairobi, 1985 jul. 25-29. Geneva: WHO; 1987. Disponível em: http://www.who.int/en/.

2. MIASSO, Al; GROU, CR; CASSIANI, SH de; SILVA, AEBC; FAKIH, FT. Erros de medicação: tipos, fatores causais e providências tomadas em quatro hospitais brasileiros. Rev. Esc. Enferm. USP. 2006; 40:524-532. Disponível em: http://dx.doi.org/10.1590/ S0080-62342006000400011

3. WHO. Promoting rational use of medicines: core components. September 2002. Geneva. Disponível em: http://apps.who.int/ medicinedocs/pdf/h3011e.pdf

4. CONSELHO FEDERAL DE FARMÁCIA. Outros CIM no Brasil. Brasília: Conselho Federal de Farmácia; 2012 [Atualizada em 20 de junho de 2012; acesso em 20 de janeiro de 2014]. Disponível em: http://cff.org.br/pagina. php?id=213\&titulo=Outros + CIM+no+Brasil

5. VIDOTTI, CCF; HELEODORO, N; ARRAIS, P; HOEFLER, R; MARTINS, R; CASTILHO, S. Centro de informação sobre medicamentos: análise diagnóstica do Brasil. Brasília: Conselho Federal de Farmácia, Organização Pan-Americana da Saúde, 2000. 72 p.

6. VIDOTTI, CCF; HOEFLER, R; SILVA, EV; BERGSTEN-MENDES, G. Sistema Brasileiro de Informação sobre Medicamentos - Sismed. Cad. Saúde Pública. 2000; 16(4):1121-1126. Disponível em: http://www.scielo.br/scielo.php?script=sci_arttext\&pid=S0102311X2000000400030\&lng=pt\&nrm=iso\&tlng=pt DOI: http://dx.doi.org/10.1590/S0102-311X2000000400030.

7. BRASIL. MINISTÉRIO DA SAÚDE. Gabinete do Ministro. Portaria N².647, de 4 de novembro de 2013. Institui a Rede Brasileira de Centros e Serviços de Informaação sobre Medicamentos (Rebracim). Disponível em: http://www.paho.org/BRA/images/ stories/BRA02C_2013/port_gm_2.647_13.pdf.

8. SOUSA, AB; SAKAI, MC. Centro de Informação sobre Medicamentos. In: STORPIRTIS, S; MORI, ALPM; YOCHIY, A; RIBEIRO, E; PORTA, V. Farmácia clínica e atenção farmacêutica. Rio de Janeiro: Guanabara Koogan, 2007; 237-250.

9. ORGANIZACIÓN PANAMERICANA DE LA SALUD. Centros de Información de Medicamentos: Una Estrategia de Apoyo al Uso Racional de Medicamentos. 1995. Santo Domingo: Informe de Grupo de Trabajo Regional, OPS.

10. KINKY, DE; ERUSH, SC; LASKIN, MS; GIBSON, GA. Economic impact of a drug information service. Ann. Pharmacother. 1999; 33:11-16.

11. BRASIL. MINISTÉRIO DA SAÚDE. Secretaria de Ciência, Tecnologia e Insumos Estratégicos. Departamento de Assistência Farmacêutica e Insumos Estratégicos. Cuidado Farmacêutico na atenção básica. 1.ed.rev. Brasília: Ministério da Saúde, 2015. [Caderno 2]

12. REBRACIM. Rede Brasileira de Centros e Serviços de Informação sobre Medicamentos - Rebracim. Brasília: Organização Pan-Americana de Saúde. [acesso em 27 de set. 2015]. Disponível em: http:// www.paho.org/bra/index.php?option =com_content \&view=article \&id=1750\%3 Arede-brasileiracentros-servicos-informacao-sobre-medicamentos-rebracim\&catid=755\%3 Abra-principal\&ltemid=876. 13. MÜLLEROVÁ, H; VLCEK, J. European drug information centres survey of activities. Pharm. World Sci., 1998; 20(3):131-5. Disponível em: http://www.ncbi.nlm.nih.gov/pubmed/9618737.

14. KARNATAKA DRUG INFORMATION AND RESEARCH CENTER. Karnataka State Pharmacy Council. Karnataka Drug Information \& Research Center. Disponível em: http://www.karnatakadruginfo.com. 